Revue des patrimoines

$42 \mid 2020$

Imagerie numérique et patrimoine culturel :

représentation et transmission des connaissances

\title{
Le numérique au service de l'exceptionnel à la bibliothèque du Service historique de la Défense : le rouleau de l'ordre de bataille de Vélez-Málaga
}

Digital medias at the service of exceptional events at the Historical Service of

Defence library: The Order of the Battle of Malaga roll

Constance de Courrèges d'Agnos, Jean-François Dubos et Sylvie Legrosse

\section{(2) OpenEdition}

Journals

Édition électronique

URL : http://journals.openedition.org/insitu/27403

DOI : 10.4000/insitu.27403

ISSN : 1630-7305

Éditeur

Ministère de la Culture

Référence électronique

Constance de Courrèges d'Agnos, Jean-François Dubos et Sylvie Legrosse, «Le numérique au service de l'exceptionnel à la bibliothèque du Service historique de la Défense : le rouleau de l'ordre de bataille de Vélez-Málaga », In Situ [En ligne], 42 | 2020, mis en ligne le 12 juin 2020, consulté le 12 janvier 2021. URL : http://journals.openedition.org/insitu/27403; DOI : https://doi.org/10.4000/insitu.27403

Ce document a été généré automatiquement le 12 janvier 2021.

In Situ Revues des patrimoines est mis à disposition selon les termes de la licence Creative Commons Attribution - Pas d'Utilisation Commerciale - Pas de Modification 4.0 International. 


\title{
Le numérique au service de l'exceptionnel à la bibliothèque du Service historique de la Défense : le rouleau de l'ordre de bataille de Vélez-Málaga
}

\author{
Digital medias at the service of exceptional events at the Historical Service of \\ Defence library: The Order of the Battle of Malaga roll
}

Constance de Courrèges d'Agnos, Jean-François Dubos et Sylvie Legrosse

1 Institution majeure dans le paysage patrimonial français, le Service historique de la Défense (SHD) est un service à compétence nationale du ministère des Armées, créé en $2005^{1}$. Il réunit au sein d'un même service, comptant dix implantations, un important centre d'archives (450 km linéaires), un département dévolu à la recherche en histoire militaire et à la gestion de la symbolique de la Défense (insignes, emblèmes, etc.) et la première bibliothèque d'Europe en histoire militaire, riche d'un million de documents.

2 Au printemps 2016, la bibliothèque du SHD a pu faire l'acquisition d'un document exceptionnel au terme d'une opération inédite de mécénat participatif, l'ordre de bataille de Vélez-Málaga. Manuscrit de près de six mètres de longueur, entièrement en couleur, réalisé vers 1715-1720, il constitue un témoignage unique, tant par sa forme que son contenu, d'une page importante de l'histoire de la Marine. Si l'acquisition de ce document atypique et précieux était un objectif de la bibliothèque du SHD, son entrée dans une collection publique n'aurait su constituer à elle seule un aboutissement. En effet, si l'enrichissement et la conservation du patrimoine collectif constituent des axes forts de l'activité de la bibliothèque, la communication et la valorisation de ses collections sont tout aussi prépondérantes. Par son caractère atypique, ce document nécessite la conjonction antinomique de deux impératifs bien connus dans le domaine patrimonial : celui de sa conservation matérielle et celui d'une médiation d'autant plus importante que son appréhension est complexe. Dès lors, le recours aux techniques 
numériques est rapidement apparu comme le moyen permettant la conciliation des opposés, au profit d'usages différents².

3 L'examen, à travers l'exemple d'un document d'exception, de la relation entre patrimoine et numérique au triple prisme de la médiation, de la conservation et de l'exploitation scientifique, ne manque pas d'interroger des pratiques professionnelles et des approches dont il convient de prendre en compte les limites. En quoi l'imagerie numérique constitue-t-elle un apport déterminant, tant outil pour les professionnels que medium pour les usagers, et quelles possibilités offre-t-elle au regard des évolutions technologiques? C'est ce questionnement, auquel l'expérience a commencé d'apporter des éléments de réponse, que l'on se propose de partager.

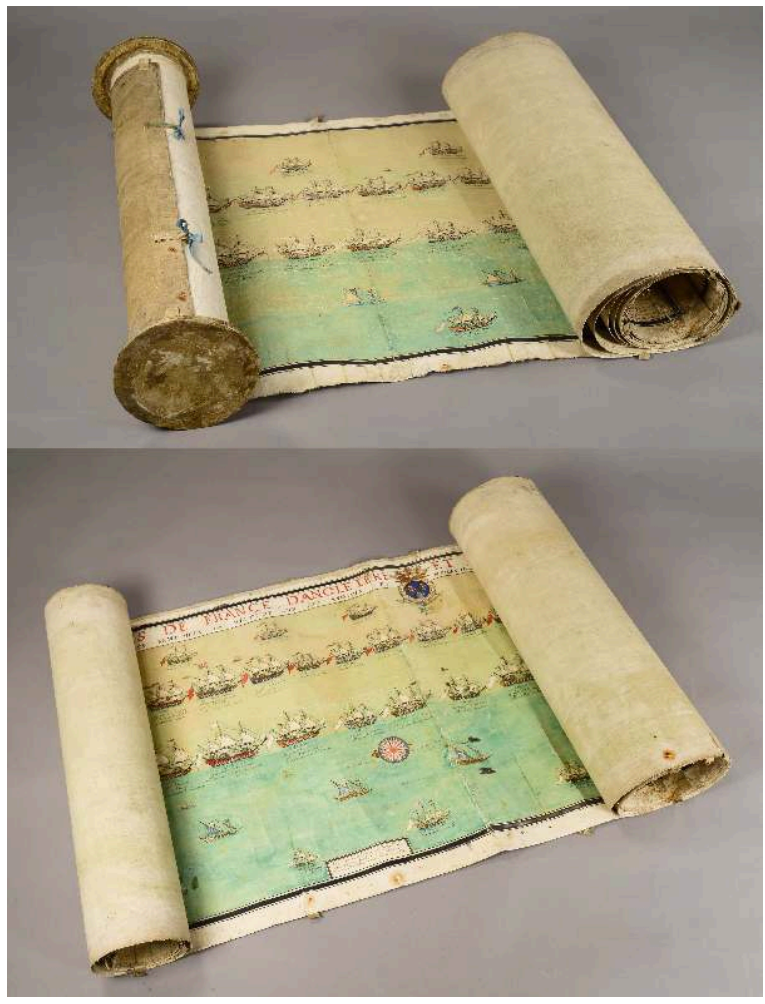

Rouleau de l'ordre de bataille de Vélez-Málaga.

Photo : D. Viola / cliché : Service historique de la Défense.

\section{L'imagerie numérique, outil et plus-value de médiation}

La difficulté d'offrir une médiation appropriée à un document hors norme a été en partie palliée par l'utilisation de l'imagerie numérique. Loin de n'être qu'une ampliation technologique du document, elle en permet une approche originale au bénéfice du plus grand nombre.

\section{Du cadre à l'écran : exposer le rouleau de Vélez-Málaga}

Si certains sujets d'exposition en bibliothèque peuvent se passer de l'apport de l'outil numérique en matière de médiation et se contenter d'une formule que l'on pourrait qualifier de "classique», d'autres nécessitent a contrario les apports permis par la 
technique en raison de leur nature, de la typologie de l'objet exposé ou encore de la conjugaison de facteurs thématiques ou documentaires ${ }^{3}$.

6 Au-delà même de la problématique propre à chaque exposition et au parti-pris scientifique de son commissariat, l'intelligibilité des documents et les questions qu'ils soulèvent en termes d'appréhension dès leur sélection sont au cœur des préoccupations des concepteurs des expositions ${ }^{4}$. Cette réflexion est d'autant plus poussée que le ou les documents concernés sont hors norme. C'est le cas pour le rouleau de l'ordre de bataille de Vélez-Málaga ${ }^{5}$, manuscrit de trois siècles et aux dimensions atypiques. Les visiteurs, dont certains ont déjà eu connaissance du document à l'occasion de la campagne de mécénat qui s'est déroulée entre mai et juillet 2016, s'attendent à découvrir et à être confrontés à un document exceptionnel, que l'apport numérique ne doit pas dévoyer ou supplanter.

\section{Pour une communication appropriée}

7 L'utilisation de moyens multimédias est apparue rapidement comme une solution avantageuse en matière de communication, les moyens techniques donnant la possibilité de déployer l'exposition selon une autre dimension. Car si la question de la pertinence de l'utilisation du numérique se pose en fonction de l'objectif visé, les contraintes induites par le caractère exceptionnel de l'élément-phare de l'exposition trouvent dans le numérique une solution globale. Sa mise en œuvre offre à la fois une entrée en matière et un approfondissement autour d'un seul et même support, dont les informations peuvent être, selon le choix du visiteur, explorées, survolées, lues, regardées, décryptées.

8 Deux dispositifs numériques ont été imaginés autour du rouleau, suite à la rencontre de spécialistes de la médiation culturelle numérique, proposant des angles d'approche différents. La richesse de ces rencontres a permis d'identifier plusieurs besoins. Ainsi s'est rapidement posée la question de l'immersion : le sujet étant centré sur le rouleau, il paraissait important de projeter le visiteur au-delà de la représentation graphique en deux dimensions. De la même façon que plusieurs chapitres d'exposition permettaient de resituer la bataille de Vélez-Málaga dans son contexte, l'outil numérique devait permettre d'immerger le visiteur dans un moment historique méconnu. Un dispositif sonore, bien que projetant le visiteur dans l'ambiance d'une bataille navale, semblait insuffisant dans la mesure où, en présence d'un document éminemment graphique, l'immersion visuelle offrait quant à elle une conception dilatée du rouleau. Parallèlement à ces éléments de "mise en contexte", une borne de consultation numérique a été implantée à proximité de l'œuvre-phare. Voulue à la fois ludique et pédagogique, accessible à tous, elle devait également favoriser une interaction entre les visiteurs. La difficulté consistait pour l'équipe du commissariat et les scénographes à cerner ce qui était attendu de l'imagerie numérique. En effet, l'attrait que représente le multimédia et la disposition pédagogique mise en place à travers la borne étaient susceptibles d'entraîner une polarisation de l'attention du public sur la borne et son contenu, et non sur le centre de l'exposition, à savoir le rouleau. Le dispositif a donc été conçu comme un objet de médiation à part entière, s'insérant avec pertinence au sein d'un propos plus large. Élément important mais ne devant pas être central, la borne interactive a été voulue comme une clé de compréhension, où éléments d'information, ludiques et d'immersion ont été équilibrés et rendus complémentaires. 


\section{Les avantages de la médiation numérique}

De nombreux avantages ont été, au regard de l'expérience, mis en exergue en ce qui concerne la médiation par le biais du numérique. L'attraction, dans un premier temps. La charte graphique, la scénarisation et l'utilisation d'un fil conducteur ont opéré sur les visiteurs une attraction renforcée par la volonté pédagogique et ludique soulignée dans le propos et la conception de la borne. Plusieurs catégories de publics se sont appropriées, chacune à leur niveau, les thématiques abordées dans l'exposition, de l'enfant découvrant la Marine de l'époque moderne ${ }^{6}$ au spécialiste qui s'attache à l'architecture navale du Grand Siècle ou à la tactique employée pendant la bataille.

L'interaction et l'immersion, dans un second temps : donner au visiteur la possibilité de se projeter dans une bataille pour qu'il en perçoive la réalité, très différente de la représentation posée offerte par le rouleau ${ }^{7}$. Il est ainsi mieux perçu, dans ses spécificités. Le document, déployé sur de nouvelles dimensions, offre l'opportunité aux visiteurs, par le moyen de la médiation numérique, de pénétrer un contexte de façon distrayante sans pour autant se détacher de l'aspect scientifique et pédagogique visé par l'exposition.

11 La place et l'espace disponibles pour l'exposition ont également vu leurs limites abolies grâce à l'apport numérique. Le choix des portraits des acteurs principaux de la bataille ou la représentation des quelque 200 navires concernés n'étaient pas sans poser question aux commissaires. Exhaustivité fastidieuse ou sélection insatisfaisante ont trouvé dans l'outil numérique une réponse commune. Ainsi, l'accumulation de données a pu offrir, par leur mise en perspective, un complément précieux aux œuvres et documents originaux présentés. S'émancipant des contraintes spatiales, la borne a pu permettre de proposer des reproductions numériques de documents parfois indisponibles pour un prêt ou aux dimensions difficilement compatibles avec une exposition grand public (plans de très grandes dimensions ou médailles de petit diamètre à l'effigie des personnages principaux, par exemple).

\section{L'imagerie numérique, acteur important de la conservation}

Le souci avéré de la conservation d'un document devenu emblématique mais suscitant l'attrait et la curiosité a trouvé des éléments de réponse attendus dans le recours à l'imagerie numérique. Celle-ci a toutefois permis d'aller plus loin.

\section{Une reproduction au-delà du simple duplicata}

L'utilisation de l'imagerie numérique trouve dans le domaine de la conservation une application désormais courante. Sans qu'il soit envisagé que l'image numérique puisse remplacer le document original, elle constitue une solution de conservation, voire de préservation. Ainsi a-t-il fallu fournir de nombreuses images du document dans le cadre de la campagne de mécénat lancée en vue de son acquisition. Axée sur son aspect exceptionnel et atypique, cette campagne avait pour objectif de permettre aux potentiels donateurs de se faire une idée de l'objet et de susciter leur participation. La campagne de mécénat et de communication ne pouvait donc se passer de visuels. Grâce 
à une numérisation complète et en haute définition, laquelle a elle-même relevé du défi technique eu égard aux dimensions hors norme du document, ce dernier n'a pas pâti de l'engouement qu'il a suscité. Seuls ses avatars numériques ont été distribués, retravaillés, mis en scène, sans que le rouleau ne soit sorti de son écrin de protection. Le statut juridique du document lors de la campagne de mécénat ne le permettait d'ailleurs pas : faute d'avoir été intégralement payé à son vendeur, il en restait sa propriété. Ce n'est qu'après signature d'une promesse d'achat, elle-même liée au succès de la campagne de mécénat, que le vendeur a accepté de confier le document à des spécialistes pour qu'il soit numérisé. Sa numérisation a donc précédé son acquisition définitive, et a participé de la communication qui l'a rendue possible. Enfin, l'imagerie numérique a été utilisée pour présenter le document après son achat, sans que l'original soit systématiquement montré, ainsi que lors des recherches préparatoires en vue de l'exposition et pour accompagner diverses publications.

\section{Un témoin de l'état de conservation...}

14 L'imagerie numérique offre, par ses propriétés techniques, la possibilité de rendre compte de façon très précise de l'état de conservation d'un document à un instant donné. Ce témoignage qui, grâce aux métadonnées qui y sont associées, apporte des informations à la fois rigoureuses et circonstanciées, est un outil précieux pour la conservation, notamment préventive. Ainsi, numériser le rouleau de Vélez-Málaga a été également l'occasion de saisir son état de conservation dès son entrée dans les collections, avant toute exposition.

Rapidement, compte tenu à la fois de son caractère exceptionnel et du recours au mécénat participatif du Service historique de la Défense, il est devenu un document emblématique non seulement de la bibliothèque patrimoniale mais également du SHD, et plus largement, des services patrimoniaux du ministère des Armées. Aussi son exposition, même brève, son déroulement, même partiel, sont-ils régulièrement demandés. Si l'on ne peut accéder à toutes les demandes pour des raisons évidentes de conservation, le contrôle de l'état du document peut s'opérer de la sorte à l'aide de l'imagerie numérique, et statuer en toute connaissance de cause en fonction d'évolutions qui, potentiellement imperceptibles à l'œil nu, ne l'en impactent pas moins. Une numérisation de qualité offre donc un « jalon de conservation » qui permet de jauger efficacement d'éventuelles variations à prendre en compte.

\section{... et un « substitut » de qualité}

16 Une autre problématique rencontrée est l'éclatement géographique du SHD, qui compte dix implantations réparties sur le territoire métropolitain. Si le rouleau est conservé à Vincennes, siège du service, chacune des implantations (Brest, Caen, Châtellerault, Cherbourg, Le Blanc, Lorient, Pau, Rochefort et Toulon) s'est impliquée pour son acquisition et s'est fait localement le relais de l'opération. Montrer aux donateurs locaux l'objet à l'acquisition duquel ils avaient contribué était une évidence. En outre, toute opération de médiation des fonds et collections devant pouvoir circuler d'une implantation à l'autre, il apparaissait indispensable d'offrir à tous la possibilité de découvrir le document. Or, les nombreuses questions liées tant aux conditions de circulation du document que de son temps d'exposition, démultipliées par le nombre d'emprises concernées, n'était pas sans soulever des inquiétudes. S'est ajoutée à ces 
préoccupations une question "de taille", celle de l'espace disponible pour exposer, dans son entièreté, un document de près de six mètres de longueur. Outre la nécessaire place requise, souvent indisponible dans des locaux contraints, disposer d'un matériel d'exposition (cadre ou vitrine) répondant aux normes de conservation s'est avéré impossible.

conséquent, le substitut numérique s'est imposé comme étant la solution la plus adaptée. Une reproduction en fac-similé, bien que mettant le public face à un objet, ne manque pas de laisser insatisfait dans la mesure où les visiteurs ne peuvent appréhender le document original. L'aspect prestigieux du document et son ancienneté participent autant de l'émotion du visiteur que son contenu informatif. Le substitut numérique, bien que privant lui aussi le public de la proximité avec l'original, a paru comme plus satisfaisant, notamment par les possibilités ainsi offertes (mise en contexte sonore, animations, etc.). C'est la raison pour laquelle ce dispositif a bien été conçu comme un substitut numérique et non comme une copie: s'affranchissant de la simple reproduction du document, l'imagerie numérique compense l'indisponibilité de l'original par une réalité augmentée qui le rend plus accessible sans en corrompre la nature et l'aspect d'origine. Ce remplacement est d'autant mieux assumé que la différence d'aspect matériel, qui n'apparaîtrait pas avec un fac-similé, témoigne justement de la volonté de ne pas présenter une copie qui pourrait être perçue comme réductrice ou destinée à tromper les visiteurs.

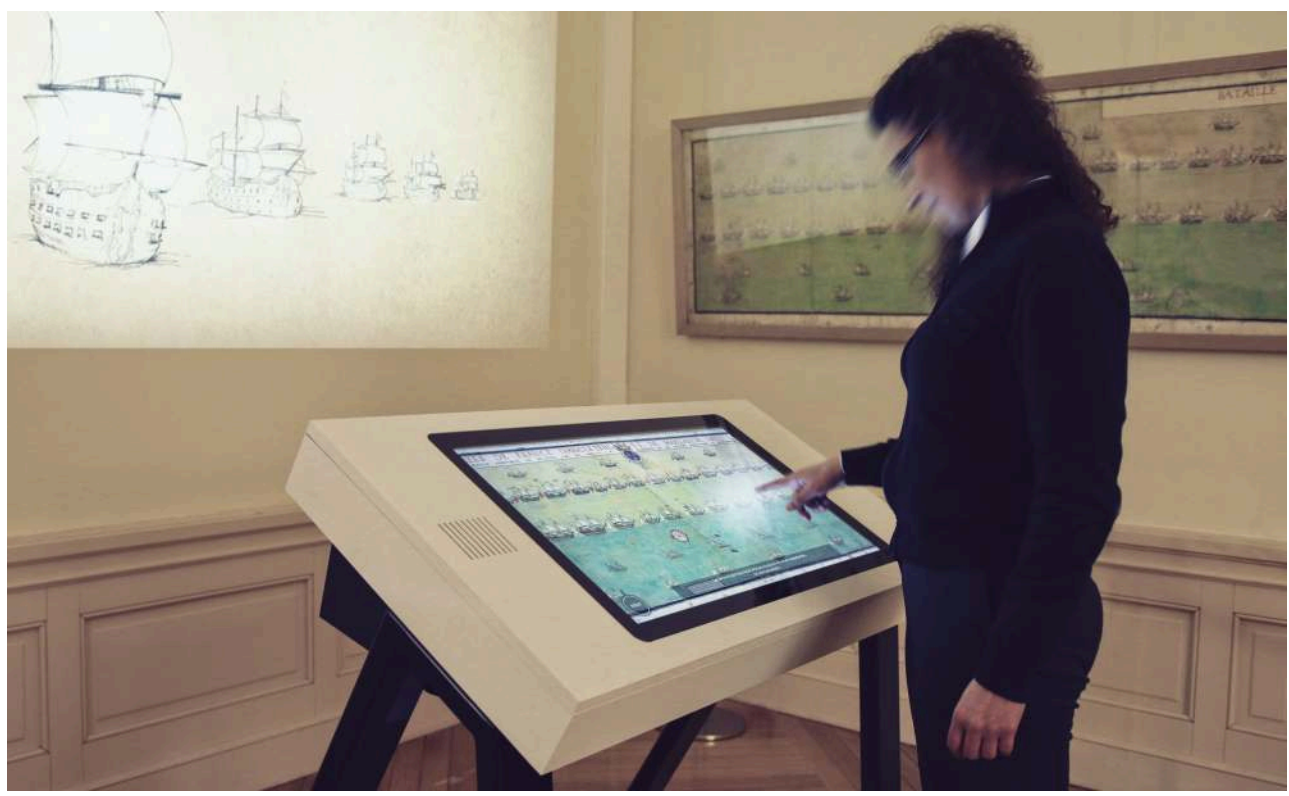

Borne interactive, support de la version numérique de l'exposition « 1704, Vélez-Málaga : quand la bataille se déroule... ».

Cliché : Fleur de papier (remerciements : Floriane Casula)

\section{Vers de nouvelles découvertes?}

Si l'accès aux documents originaux est parfois indispensable, l'image numérique compense nettement la distance établie avec ces derniers par des apports que la technologie offre aux chercheurs ${ }^{8}$. 


\section{Un regard différent sur le document}

Témoignage rare de la guerre sur mer sous l'Ancien Régime, le rouleau de l'ordre de bataille de Vélez-Málaga est apparu sur le marché accompagné d'un article qui, pour informatif qu'il soit, émanait de son vendeur. Avant même d'envisager son acquisition, il a été nécessaire d'effectuer des recherches préalables permettant d'éclaircir les nombreuses questions suscitées par les zones d'ombre du document et son aspect étonnant. Les dimensions du rouleau, la richesse et la complexité des dessins, les recherches sur les techniques de sa réalisation ont été autant d'éléments qui ont nécessité un temps long d'étude et, de facto, de consultations fréquentes du rouleau, au risque de détériorations qui, même minimes, n'en seraient pas moins fortement préjudiciables à un unicum.

Désormais conservé dans une boîte sur mesure, aux normes de conservation, et dans un magasin adapté, le document original n'est communicable qu'à la double condition que son avatar numérique n'offre pas l'accès aux informations qui pourraient faire défaut à des codicologues, chercheurs sur les pigments de l'époque ou autres spécialistes, et que les conditions de sécurité et de conservation permettent son déploiement le temps de sa consultation. Celle-ci reste donc exceptionnelle, même si la recherche sur copie numérique n'est pas exclusive: si elle permet de travailler plus longuement et à plusieurs sur le même document, elle n'interdit pas le recours à la consultation in fine du document, et pour cause : parce que l'imagerie numérique donne à voir le rouleau, elle préserve ce dernier qui sera d'autant plus disponible pour une ultime consultation physique.

\section{Croiser les informations et disséquer les données}

21 La recherche aidée par l'imagerie numérique est également porteuse de nouvelles perspectives. En effet, les moyens multimédias permettent une comparaison de certains éléments (en l'occurrence gréements, sabords, etc.) parfois bien plus poussée qu'une étude à l'œil nu, qui peut se révéler imprécise, du moins plus incertaine. Comparaisons, superpositions, déconstruction numérique de l'image sont autant de techniques pouvant alimenter la recherche. Une précision plus grande dans l'étude de certains aspects du document, possible pour plusieurs chercheurs simultanément, rend plus aisés le croisement des informations et la confrontation possible de plusieurs documents disponibles sous format numérique. De nouveaux rapprochements peuvent avoir lieu, des comparaisons de techniques, de matériaux et de contenus, à l'image de ce qui se pratique déjà depuis longtemps pour les œuvres d'art, peuvent ainsi s'appliquer et augmenter la plus-value informative du document.

\section{Un atout évident, mais aux limites perceptibles}

Si le tableau brossé ci-dessus insiste sur les atouts de l'imagerie numérique à de nombreux points de vue, il convient de n'écarter ni l'importance de la conservation matérielle d'un document original et unique, ni les limites d'un outil dont les possibilités masquent les écueils. Les conséquences à long terme d'une entreprise de numérisation, quelle qu'en soit la qualité et le respect des normes, sont encore mal connues. En outre, la nécessité de procéder éventuellement à une nouvelle 
numérisation dans quelques années, pour bénéficier des apports de technologies à l'évolution rapide, pourrait être sujette à débat. Où fixer les limites entre la "nécessité numérique " et l'excès dont l'utilité peut être discutée? L'imagerie numérique ne saurait, malgré sa contribution, occulter l'intérêt du contact visuel avec le document original. De même, elle ne doit ni le remplacer ni le reléguer. Enfin, le risque de trahir l'original par les moyens offerts par l'outil numérique reste présent. Les normes respectées et le souci scrupuleux des agents travaillant sur le document d'y être les plus fidèles possible n'éliminent toutefois pas totalement le risque d'une erreur colorimétrique, d'un rendu légèrement modifié, voire la tentation d'une retouche malheureuse. Outil et non objectif, l'imagerie numérique est un outil précieux pour les gestionnaires de collections patrimoniales, mais qui doit être utilisé à bon escient. C'est ce à quoi s'emploie la bibliothèque du Service historique de la Défense.

\section{NOTES}

1. www.servicehistorique.sga.defense.gouv.fr

2. Sur ces questions, voir DUFRÊNE Bernadette, IHADJADENE Madjid \& BRUCKMANN Denis, Numérisation du patrimoine. Quelles médiations? Quels accès? Quelles cultures?, Paris, Hermann, coll. «Cultures numériques », 2013.

3. Voir PAYEN Emmanuèle, «La bibliothèque s'expose : concevoir et réaliser une exposition ", in PAYEN Emmanuèle \& HUCHET Bernard (dir.), L'Action culturelle en bibliothèque, Paris, Éditions du Cercle de la librairie, 2008.

4. Sur cette question d'équilibre, voir notamment BENAITEAU Carole, « La conception. Imaginer l'exposition ", in BENAITEAU Carole, BENAITEAU Marion, BERTHON Olivia \& LEMONNIER Anne, Concevoir et réaliser une exposition. Les métiers, les méthodes, Paris, Eyrolles, 2012.

5. Bataille navale qui opposa la France aux Anglais dans la guerre de Succession d'Espagne, qui se déroula au sud de Málaga le 24 août 1704.

6. Voir OCTOBRE Sylvie, Culture prospective, $\mathrm{n}^{\circ} 1$, «Pratiques culturelles chez les jeunes et institutions de transmission : un choc de cultures?», 2009, p. 1-8. Disponible en ligne, https:// www.cairn.info/revue-culture-prospective-2009-1-page-1.htm [lien valide en mai 2020].

7. À ce sujet, voir VIDAL Geneviève, «La médiation numérique et les musées : entre autonomie et prescription", in GALAUP Xavier (dir.), Développer la médiation documentaire numérique, Villeurbanne, Presses de l'Enssib, 2012.

8. Cette question accompagne l'essor et le développement de l'outil numérique, comme en témoignent les actes du colloque organisé en 1996 à la BnF par l'École nationale du patrimoine : Patrimoine et multimédia: le rôle du conservateur, Paris, École nationale du patrimoine / La Documentation française, 1997. 


\section{RÉSUMÉS}

La bibliothèque du Service historique de la Défense, service à compétence nationale du ministère des Armées, a acquis en 2016 par voie de mécénat participatif un document exceptionnel. Entièrement manuscrit, le rouleau de l'ordre de bataille de Vélez-Málaga (vers 1715-1720) a rapidement fait l'objet d'une numérisation, tant pour des raisons de conservation que de médiation. L'imagerie numérique s'est imposée comme un moyen intéressant de médiation offrant une vraie plus-value scientifique et pédagogique à ce document si particulier. Outre sa valorisation, la numérisation du document offre des possibilités intéressantes du point de vue de la conservation, en proposant un substitut et en témoignant de l'état de conservation du rouleau. C'est enfin la recherche que sert l'imagerie numérique, en permettant un regard autre sur le document. Le croisement des informations et des données, rendu possible par le numérique, apporte également de nouvelles perspectives de recherche, même s'il faut être conscient des limites de l'imagerie numérique, quel que soit son domaine d'application. Car si l'imagerie numérique est un outil aux possibilités exceptionnelles, elle ne saurait être utilisée à bon escient sans une réflexion sur ses limites.

The library of the Historical Service of Defence, a nationwide service of the Ministry of Armed forces, purchased an exceptional document thanks to a crowd-funding campaign in 2016. This 18th century manuscript, The Order of the Battle of Malaga roll (c. 1715-1720) was rapidly digitized for both mediation and conservation purposes. Digital imaging then emerged as an interesting way of mediation, offering a real scientific and pedagogic added value to such a document. Besides interpretation, digitisation allows interesting possibilities in terms of preservation, providing both a high-quality surrogate and a testimony of the manuscript's state of conservation. Finally, digital imagery helps historical research offering a new point of view on the document. Digitisation has made the crossover of information and data possible, while underlining the limits of digital imagery, regardless of its field of application. Although digital imaging is a tool with exceptional potential, it cannot be used wisely without thinking about its limits.

\section{INDEX}

Mots-clés : France, Service historique de la Défense, bibliothèques spécialisées, patrimoine culturel, conservation et restauration, histoire navale, médias numériques

Keywords : France, Historical Service of Defence, specialised libraries, cultural heritage, conservation and restoration, naval history digital media

\section{AUTEURS}

\section{CONSTANCE DE COURRÈGES D'AGNOS}

Aspirant de Gendarmerie, chargée de missions auprès du chef du département de la bibliothèque du Service historique de la Défense (SHD, ministère des Armées). constance.de-courreges-d'agnos@intradef.gouv.fr 


\section{JEAN-FRANÇOIS DUBOS}

conservateur en chef, chef du département de la bibliothèque du Service historique de la Défense (SHD, ministère des Armées).

jean-francois.dubos@intradef.gouv.fr

\section{SYLVIE LEGROSSE}

Gestionnaire de collections au sein de la division des Livres anciens et Manuscrits du département de la bibliothèque du Service historique de la Défense (SHD, ministère des Armées). sylvie.legrosse@intradef.gouv.fr 